\title{
Patient Education Level and Utilization of Internet Resources by Patients in Orthopedic Hip and Knee Consultations
}

\author{
Kevin Koo, Chris Farlinger, Samuel Johnson, Khalid A. Syed \\ Division of Orthopedic Surgery, University of Toronto, Toronto, Canada \\ Email: kevin.koo@utoronto.ca, chris.farlinger@utoronto.ca, Samuel.Johnson@uhn.ca, khalid.syed@uhn.on.ca
}

Received November 17, 2012; revised December 20, 2012; accepted December 28, 2012

\begin{abstract}
Introduction: Internet has become an increasingly popular source of reference for patients to learn about their medical problems. It is easily accessible, and a large number of uncensored information is available online written from various sources and perspectives. However, the role of internet and its impact on patient's care and understanding of the disease remains unclear. The purpose of this study is to evaluate the role and effect of internet use for patients seeking consultation for hip and knee arthritis. More specifically, the relationship between patient's education level, internet use, motives for doing background readings, perception of internet information, and reactions to the available information will be studied. Method: Patients seeking orthopaedic consultation for knee or hip arthritis at the Toronto Western Hospital were identified and invited to fill out a questionnaire on their first visit. The questionnaire was designed to assess the patients' pre-consultation reading habits, their use of internet, and their reaction to what they have read on the internet. The questionnaire also included questions about the respondent's background. Results: In comparing patients holding college/university degree (CU) with patients having no college/university education (NoCU), the CU group were associated with increased internet use (CU vs. NoCU: $71.0 \%$ vs. $48.3 \% ; p<0.01$ ) and background reading (CU vs. NoCU: $82.2 \%$ vs. $17.8 \% ; p<0.001$ ) prior to consultation; fewer incidence of anxiety following internet use (CU vs. NoCU: $29.9 \%$ vs. $53.6 \% ; p<0.05$ ); and higher rates of decisions influenced by internet use (CU vs. NoCU: $20.8 \%$ vs. 3.6\%, p $<0.05$ ). Internet users demonstrated a higher confidence in gathering and understanding medical information (Internet users vs. non-internet users: $6.59 \pm 2.05$ vs. $5.03 \pm 2.78 ; p<0.001$ ) and rated the accuracy of information on internet at $7.18 \pm 2.01$ ( $\max =10)$. Conclusion: Internet use can influence patient's treatment decision, anxiety level, and understanding of their disease. Caregivers must recognize the growing trend of internet use and should counsel and educate their patients appropriately based on what they have read to help them accurately appreciate the nature of their disease.
\end{abstract}

Keywords: Patient Education; Internet Use; Orthopedic Hip; Knee Consultations

\section{Introduction}

The anticipation of an operation can cause patients and their family to experience undue stress and anxiety. Previous reports indicate that up to $60 \%$ of patients undergoing elective procedures will experience some form of anxiety prior to surgery [1]. Patient anxiety is an important consideration as it directly influences the course of their treatment [2-4]. Moreover, preoperative anxiety has been shown to contribute directly to surgical outcomes [5,6]. Elevated levels of anxiety and stress have been shown to increase post-operative pain, lengthen hospital stays, prolong patient recovery time and attenuate adherence to hospital discharge regimes [6]. To further complicate matters, patients who continue to be anxious postoperatively have a higher incidence of post-operative complications and return hospital visits [6].
Of the controllable factors, patient confidence and education regarding the procedure have been shown to have a direct correlation to improved outcomes [7]. Previous research has shown that patients who are provided with appropriate information preoperatively regarding the surgery, anticipated recovery, and potential discharge dates have decreased stress and anxiety [7]. Furthermore, patient's receiving accurate information prior to surgery augment their ability to manage their own care, attenuate recovery time and improve adherence to hospital discharge plans [6,7]. Thus, patient education leading to decreased stress preoperatively could have significant benefits on patient outcomes.

In an ideal practice, patients are educated by their physicians on the nature of their disease and the risks and benefits of the available treatments. However, with increasing demand of orthopaedic surgeons by the com- 
munity and the large number of patients that are seen in a clinic, time is not always afforded to patients to appropriately educate them on their disease or even to answer their questions. Most of the time, the onus of patient education rests upon the patient themselves. The motivetion for patient to understand their own disease is factored by the ease of resource accessibility, the complexity of the language used by the available resources, and the patients' perception of the severity of their ailment. Based on these factors, it is not hard to imagine the difference in patient's motivation to learn will vary between different patient demographics, for example, age or education level.

The educational material available to patients is almost limitless with the advent of the Internet. The Internet is able to provide patients with easy access to information regarding any condition. Whereas patients were once required to seek advice from a healthcare professional they are now able to access a plethora of information without leaving the house. The fact that the Internet is devoid of all informational boundaries however complicates matters, as the information patients receive is not regulated. For example, a common patient complaint is hip or knee arthritis and while many reputable and peer-reviewed orthopedic websites exist, there are just as many nonreviewed sites providing misleading and false information. Consequently, while the Internet is meant to help educate individuals, its impact on patient care remains unclear. Other studies have attempted to evaluate the accuracy of internet for patients in various other surgical specialties. All have concluded that the internet is not a dependable source of accurate information for patients, to improve our understanding the effects of Internet on patients, it is needed, first, to identify factors contributing to avoid misleading information and appropriate one. One of such factor is educational background of the patient. It has been wildly acknowledged that educational background facilitate treatment outcome [19]. However, it remain unclear whether a certain educational background is necessary to filter through the useless information to find appropriate resources the actual effect this information has on patients remained largely unstudied [8-11]. The purpose of this investigation is thus to assess the role of the Internet for patients seeking consultation for hip and knee arthritis and to assess their feelings of stress and anxiety regarding this information. Furthermore, this study will assess the difference in internet use and the patients' reaction to what they have read across different education level.

\section{Materials and Methods}

Between the periods of February 2009 to October 2009, patients who were seen in the Toronto Western Hospital Orthopaedic clinics for either a hip or knee arthritis con- sults were invited to complete a questionnaire regarding their use of the internet for their current hip or knee problem. The surveys were filled out anonymously, and study subjects who were over 18 years old, read, write and understand English with no prior history of any orthopaedic consultations were invited into the study. Among the 201 patients who agreed to participate in the study, 165 surveys were completed and returned.

The first component of the survey helped determined patient's highest education level, and this was separated into elementary school, middle school, secondary school, post-secondary certification, college, university, and graduate school. For the purpose of this study, patients were categorized under higher education level (college, university or graduate school), or less than college education (elementary, middle, secondary, or certification school).

The second question of the survey questioned the patients of their background reading habit prior to their visit and the type of reading materials they used. The response formats were in the form described below;

Regarding the background reading on the condition prior to clinic visit.

YES or NO

If "yes" list of the items utilized.

Internet, Magazines, Pamphlets, TV, Film, Newspaper and Other sources.

Additionally, patients were asked to assess the information they have gained from the internet using a 10 point Likert scale on accuracy, appropriateness, reliability, clarity, and ease of finding information.

The response formats were in the form described below;

Regarding assessment of information received from the internet.

\begin{tabular}{cc}
\hline Accuracy & $1-10$ with 1 as LEAST and 10 as MOST \\
\hline Appropriateness & $1-10$ \\
Reliability & $1-10$ \\
Clarity & $1-10$ \\
$\begin{array}{c}\text { Ease of Finding } \\
\text { Information }\end{array}$ & $1-10$ \\
\hline
\end{tabular}

For patients that did not use the internet as a source of background reading, they were asked in a separate question for their reasons of not using the internet. They were asked to choose from one of the following choices: 1) No computer access; 2) no internet access; 3) lack of knowledge with the use of the internet; 4) did not know such medical information were available on the internet; 5) cost; 6) prefer to get information from the doctor.

Regarding the Reason for not using internet.

The response formats were in the form described be- 
low;

A blank space for placing a check mark in front of the 6 choices provided.

\begin{tabular}{c} 
No computer access \\
No internet access \\
Don't know anything about internet \\
Didn't know could get such information on internet \\
Too expensive \\
Prefer to get information from doctor \\
\hline
\end{tabular}

Regarding the patients that have used the internet, description of their motivation was obtained by the patients placing a check mark in front of the area of their curiosity.

The topics of interest were.

\begin{tabular}{cc}
\hline 1 & General information about arthritis \\
2 & Treatment options (surgical vs no surgical) \\
3 & Ways to cope with it \\
4 & Recovery period after surgery and time needed to return to \\
5 & normal level of activity \\
6 & Complications of Surgery \\
7 & Oroups \\
\hline
\end{tabular}

For patients that have used the internet, they were asked to describe their motivation to use internet for background reading. Patients were also inquired regarding their reactions to what they have read online in a series of "yes" and "no" type of questions which include: 1) increased confusion; 2) more hopeful; 3) more anxious; 4) discussed with doctor about what they have read on the internet; 5) influence on treatment decisions; 6) learned of new treatments; 7) learned of clinical trials.

When patients were asked to rate their confidence level in gathering and understanding medical information prior to their consult on a scale of 1 to 10 , internet users were found to be more confident $(6.59 \pm 2.05)$ than noninternet users $(5.03 \pm 2.78)(p<0.001)$.

Finally, the patients were asked about how much information they desired about their condition. They were asked to choose from one of the following three choices: 1) I want only the information needed to take care of myself properly; 2) I want additional information only if it is good news; 3) I want as much information as possible, good or bad.

\section{Data Analysis}

The Student $t$-test was used to assess for statistical sig- nificance in patient's confidence in gathering and understanding medical information between the two groups of education levels. A chi-square test was utilized to measure significant difference in patient's background reading, internet use, and their reactions to what they have read on the internet based on their education levels. In cases where an expected frequency of an occurrence totaled less than 10, a Fisher-exact test was employed to determine the exact probability. The minimum requirement for statistical difference to be considered significant was set at $p<0.05$.

\section{Results}

Of the 201 patients invited to participate in the study, 165 (82\%) of them completed and returned the survey. With respect to the participants' education level, 107 (64.8\%) of the patients held at least a college or university degree.

Participants were asked about background reading prior to their first consultation in the survey, 121 (73.3\%) of the total participants have indicated that they have read about their current condition of interest, and 105 (86.8\%) patients who did background reading utilized the internet as one of their primary resource. A summary of patients' background reading resources is provided in Table 1. A total of 60 (36.4\%) of the participants did not use internet to do background reading prior to their visit. There was a difference in patients' education level among those who did background reading and those who used internet as a resource (Tables $\mathbf{2}$ and 3, respectively). More specifically, a greater proportion of patients with post-secondary education did prior readings $(p<0.001)$ and utilized internet $(p<0.01)$ compared to those who do not have a college or university degree.

Among the 105 internet users in this study, they were asked the types of information they sought on the internet (Table 4). 65 (61.9\%) used the internet to get General Information of the condition while 92 (87.6\%) were more interested in knowing the treatment options available. 71 (67.6\%) obviously not wanting surgery as treatment

Table 1. Background reading sources (of those that did background reading).

\begin{tabular}{cc}
\hline Source & Total (\%) \\
\hline Internet & $105(86.8 \%)$ \\
Magazines & $17(14.0 \%)$ \\
Pamphlets & $19(15.7 \%)$ \\
TV & $10(8.3 \%)$ \\
Film & $0(0 \%)$ \\
Newspaper & $11(9.1 \%)$ \\
Others & $26(21.5 \%)$ \\
\hline
\end{tabular}


Table 2. Patient education level in background reading.

\begin{tabular}{cccc}
\hline Education & $\begin{array}{c}\text { With background } \\
\text { reading }\end{array}$ & $\begin{array}{c}\text { No background } \\
\text { reading }\end{array}$ & Total \\
\hline $\begin{array}{c}\text { Elementary- } \\
\text { Certificate } \\
\begin{array}{c}\text { College or } \\
\text { higher }\end{array}\end{array}$ & $33(56.9 \%)$ & $25(43.1 \%)$ & 58 \\
Total & $88(82.2 \%)$ & $19(17.8 \%)$ & 107 \\
\hline
\end{tabular}

Table 3. Patient education level in internet users.

\begin{tabular}{cccc}
\hline Education & Internet use & No internet use & Total \\
\hline $\begin{array}{c}\text { Elementary- } \\
\text { Certificate }\end{array}$ & $28(50.0 \%)$ & $30(50.0 \%)$ & 58 \\
College or higher & $77(71.0 \%)$ & $30(29.0 \%)$ & 107 \\
Total & 105 & 60 & 165 \\
\hline
\end{tabular}

Table 4. Type of information interested by internet users.

\begin{tabular}{cc}
\hline Type of information & Total (\%) \\
\hline General information & $65(61.9 \%)$ \\
Treatment options & $92(87.6 \%)$ \\
Ways to cope with it & $71(67.6 \%)$ \\
Recovery period after surgery & $60(57.1 \%)$ \\
Complications of surgery & $56(53.3 \%)$ \\
Chatrooms/discussions groups/online groups & $3(2.9 \%)$ \\
Other & $7(6.7 \%)$ \\
\hline
\end{tabular}

wanted to know the ways to cope with it. Those interested in the surgical option 60 (57.1\%) were interested in the Recovery period after surgery, 56 (53.3\%) in the complications of the surgery and finally 3 (2.9\%) and Other 7 (6.7\%) were interested in discussions in Chat rooms and on-line Arthroplasty Forums. (Table 5) shows the reasons for information search on the internet. In this group, 86 (81.9\%) internet users used the internet to learn about the disease and to investigate the various treatment options. 57 (54.3\%) search for options to help coping with the disease and 13 (12.4\%) for other reasons. Internet users were also asked to rate on a scale of 1 to 10 their perception on the internet's information based on its accuracy, appropriateness, reliability, clarity, and ease of search (Table 6).

Internet users were then asked to describe their reactions to what they have read on the internet (Table 7). Overall, 22 (21.0\%) users had increased confusion, 33 (31.4\%) users had increased anxiety, and 34 (32.4\%) found themselves more hopeful following background reading on the internet. Additionally, 20 (19.0\%) patients in this category indicated that their reading on the inter-
Table 5. Reasons for information search on internet.

\begin{tabular}{cc}
\hline Reasons & Total (\%) \\
\hline To understand the disease better & $86(81.9 \%)$ \\
To investigate treatment options & $86(81.9 \%)$ \\
To help in coping with the disease & $57(54.3 \%)$ \\
Others & $13(12.4 \%)$ \\
\hline
\end{tabular}

Table 6. Information rating.

\begin{tabular}{cc}
\hline Rating & Average $( \pm$ SD) \\
\hline Accuracy & $7.18( \pm 2.01)$ \\
Appropriateness & $7.11( \pm 2.06)$ \\
Reliability & $6.78( \pm 2.12)$ \\
Clarity & $6.94( \pm 1.99)$ \\
Ease of finding & $7.60( \pm 2.26)$ \\
\hline
\end{tabular}

Table 7. Patients' reactions following internet use.

\begin{tabular}{cc}
\hline & Total (\%) \\
\hline Increased confusion & $22(21.0 \%)$ \\
More anxious & $33(31.4 \%)$ \\
More hopeful & $34(32.4 \%)$ \\
Discussed with doctor about internet information & $30(28.6 \%)$ \\
Internet influenced decision & $20(19.0 \%)$ \\
Learned of new treatments & $38(36.2 \%)$ \\
Learned of clinical trials & $18(17.1 \%)$ \\
\hline
\end{tabular}

net has influenced their decision on the treatment, and only 30 (28.6\%) patients discussed with their doctor regarding what they have read on the internet. In compareing the two groups of patients based on their education level, patients without a post-secondary school degree had a slightly higher percentage of increased confusion and gained more hope after reading the internet, and had a smaller percentage of individuals who discussed what they have read with their doctors; however, these differences were not statistically significant (Table 8). Conversely, post-secondary graduates were more likely to be influenced in their treatment decision based on what they have read on the internet when compared to patients with lower educations $(p<0.05)$ (Table 8). More importantly, anxiety following internet reading was greater in respondents with no college or university education than those with a degree $(p<0.05)$.

Among the 60 non-internet users, they were asked for their reason not to use the internet (Table 9). Forty-three $(71.7 \%)$ of the non-internet users preferred to receive 
their information directly from their physician, and none of them attributed their lack of internet use to the high cost of computer or internet access.

When the 165 patients were asked about who the decision maker should be in a doctor-patient relationship, 40 (24.2\%) responded doctors only, 114 (69.1\%) responded both doctor and patient, and 11 (6.7\%) responded patients only. Finally, participants were also asked about how much information they would like to know about their condition (Table 10), and 135 (81.5\%) of the respondents indicated that they would like to know as much information as possible, good or bad.

\section{Discussion}

As of December 2009, over 250 million (76.2\%) North Americans are internet users and $26.6 \%$ of the world's population has access to the internet [13]. The number of internet users continues to grow on a yearly basis, and with over 1.8 billion internet users around the world [13], the amount of information that are shared on the world wide web are enormous. In this study, $63.6 \%$ of the participants have indicated they have used the internet to

Table 8. Patients' reaction and education levels.

\begin{tabular}{ccc}
\hline & Elementary-Certificate & $\begin{array}{c}\text { College or } \\
\text { higher }\end{array}$ \\
\hline Increased confusion & $6(21.4 \%)$ & $16(20.8 \%)$ \\
More anxious & $15(53.6 \%)$ & $23(29.9 \%)$ \\
More hopeful & $11(32.4 \%)$ & $23(67.6 \%)$ \\
$\begin{array}{c}\text { Internet influenced } \\
\text { decision }\end{array}$ & $1(3.6 \%)$ & $16(20.8 \%)$ \\
\hline
\end{tabular}

Table 9. Reasons for not using internet.

\begin{tabular}{cc}
\hline Reasons & Total (\%) \\
\hline No computer access & $7(11.7 \%)$ \\
No internet access & $2(3.3 \%)$ \\
Didn't know anything about internet & $9(15.0 \%)$ \\
Didn't know could get such information on internet & $10(16.7 \%)$ \\
Prefer to get information from doctor & $0(0 \%)$ \\
\hline
\end{tabular}

Table 10. Desired information.

\begin{tabular}{cc}
\hline & Total (\%) \\
\hline I want only the information needed to take care of \\
myself properly & $28(17.0 \%)$ \\
I want additional information only if it is good news & $2(1.2 \%)$ \\
I want as much information as possible, good or bad & 135 \\
\hline
\end{tabular}

learn more about their current arthritis. While there are many quality academic or organizational websites that are geared towards patient education, it is difficult for patients with no experience in retrieving medical information gather all the accurate facts in their condition. With free uncontrolled sharing of information on the web, patients can easily be presented with misleading or inaccurate information on the internet. Although we can notforbid patients from learning about their disease on the internet, we can try to understand the effect internet reading can have on patients.

The internet has become the most popular forum to do background reading for patients enrolled in our study, and this finding is consistent with other studies that have looked at patients' internet use [10,14,15]. Of the 121 patients that have done background reading, $86.8 \%$ of the participants have relied on the internet. We have also demonstrated that patients with post-secondary education were more likely to do background reading and to use the internet when compared to those with no post-secondary degrees. Lower socioeconomic status and education has been attributed to more patient co-morbidities [18,19], and although a limitation of this study is that we were not able to examine the reliability and validity of the measures, the trend we have demonstrated here shows that those with higher level of education are more proactive in caring for their own ailments, have the means to access internet resource for reading, and more willing to learn about the cause and treatments of their disease.

More interestingly, different education levels may result in different reactions from the patients. Although education level was not a factor in causing confusion following internet reading, individuals with no postseconddary degrees were shown to have more anxiety after reading when compared to their counterpart. This may be due to the difficulty that the patients in this category have in understanding the medical information on the internet. Additionally, a higher proportion of patients with post-secondary education have their made their treatment decisions based on what they have read on the web prior to their consultations. Although the trend demonstrated in this study shows that internet reading has generally helped individuals with higher education with patient anxiety, internet reading may actually be harmful for this group of individuals. Some internet sites are more persuasive than others, and thus, becomes a potentially dangerous resource that can influence patients negatively. If an internet site wrongfully changes the patient's perception of their condition and treatments, it becomes harder for the physicians to convince the patients to consider and accept alternative treatments. Nothing is worst than denying a patient with no indications of an operative treatment when they are insistent that this is the only management they are seeking.

We have shown that internet users have a higher de- 
gree comfort and confidence in searching for medical information compared to non-internet users. However, we must be cautious with their comfort, as this is the patients' own perception of confidence, and it is difficult to determine whether a higher level of confidence translates into the ability to search for the most accurate websites or the ability to discern between two websites of different qualities.

Arthritis is a condition which affects mostly patients over 50 years of age-an age group in which internet and computer access was not readily accessible when they were growing up. With the increase spread of internet use around the world, the number of patients that will become reliant on the internet to learn about their disease will only increase. Our study looked at a cross-section of the current use and response to internet as a source of background reading for patients. The use of internet by patients will very much be different and unpredictable even a year from now. Practitioner must not be oblivious to the fact that their patients will now seek their information on the web in addition to their consultation.

Many studies have suggested creating guidelines when constructing a medical website to ensure the medical information is evidence-based and bias free [11,16,17]. This is an ideal solution, however difficult to enforce. We tend overlook the importance of educating the patients and anticipating the reactions that the patient will have from reading both the facts and the myths that lies within the net. In our study, $81.8 \%$ of the patients wanted to know every detail, good or bad, regarding their condition, and $71.7 \%$ of non-internet users prefer to get their medical information from their doctors. It is not enough to just advise patients to stay away from reading the information on the internet. Practitioners must properly provide resources and take the time to educate their patients and clarify questions that arises during the consultation.

In summary, the rapid expansion of internet use by patients is inevitable, and physicians must embrace their practice by effectively incorporating the use of this information to improve patient outcomes. This would mean identifying concerns that patients have from their reading, recognizing the difference in patients' reactions and response to internet information across the various domains of patients' demographics, and proactively providing patients with educational resources that can help them make smart and informed decisions.

\section{REFERENCES}

[1] A. Boker, L. Brownell and N. Donen, “The Amsterdam Preoperative Anxiety and Information Scale Provides a Simple and Reliable Measure of Preoperative Anxiety,” Canadian Journal of Anesthesia, Vol. 49, No. 8, 2002, pp. 792-798. doi:10.1007/BF03017410
[2] D. Fell, D. R. Derbyshire, C. J. Maile, I. M. Larsson, R. Ellis, K. J. Achola and G. Smith, "Measurement of Plasma Catecholamine Concentrations. An Assessment of Anxiety,” British Journal of Anaesthesia, Vol. 57, No. 8, 1985, pp. 770-774. doi:10.1093/bja/57.8.770

[3] C. Weissman, "The Metabolic Response to Stress: An Overview and Update,” Anesthesiology, Vol. 73, No. 2, 1990, pp. 308-327. doi:10.1097/00000542-199008000-00020

[4] T. M. Osborn, and N. A. Sandler, "The Effects of Preoperative Anxiety on Intravenous Sedation,” Anesthesia Progress, Vol. 51, No. 2, 2004, pp. 46-51.

[5] I. Maranets and Z. N. Kain, "Preoperative Anxiety and Intraoperative Anesthetic Requirements," Anesthesia \& Analgesia, Vol. 89, No. 6, 1999, pp. 1346-1351.

[6] C. J. Eloise, V. N. Thomas and J. Wilson-Barnet, "Patient Experiences of Anxiety, Depression and Acute Pain after Surgery: A Longitudinal Perspective,” International Journal of Nursing Studies, Vol. 42, No. 5, 2005, pp. 521-530. doi:10.1016/j.ijnurstu.2004.09.014

[7] J. Heine, S. Koch and P. Goldie, "Patients' Experiences of Readiness for Discharge Following a Total Hip Replacement," Australian Journal of Physiotherapy, Vol. 50, No. 4, 2004, pp. 227-233.

[8] A. K. Madan, C. T. Frantzides and C. E. Pesce, “The Quality of Information about Laparoscopic Bariatric Surgery on the Internet,” Surgical Endoscopy, Vol. 17, No. 5, 2003, pp. 685-687.

[9] L. C. Soot, G. L. Moneta and J. M. Edwards, "Vascular Surgery and the Internet: A Poor Source of Patient-Orientated Information,” Journal of Vascular Surgery, Vol. 30, No. 1, 1999, pp. 84-91. doi:10.1016/S0741-5214(99)70179-5

[10] N. Aslam, D. Bowyer, A. Wainwright, T. Theologis and M. Benson, "Evaluation of Internet Use by Paediatric Orthopaedic Outpatients and the Quality of Information Available,” Journal of Pediatric Orthopaedics B, Vol. 4, No. 2, 2005, pp. 129-133.

doi:10.1097/01202412-200503000-00014

[11] P. K. Beredjiklian, D. J. Bozentka, D. R.Steinberg and J. Bernstein, "Evaluating the Source and Content of Orthopaedic Information on the Internet: The Case of Carpal Tunnel Syndrome,” Journal of Bone and Joint Surgery, Vol. 82A, No. 11, 2000, pp. 1540-1543.

[12] SEO Consultants, “Top Ten Search Engines-Top 10 SEs," 2010.

http://www.seoconsultants.com/search-engines/

[13] Internet World Stats, "Internet Usage Statistics for all the Americas,” 2010. http://www.internetworldstats.com/stats2.htm

[14] G. O. Hellawell, K. J. Turner, K. J. Le Monnier and S. F. Brewster, "Urology and the Internet: An Evaluation of Internetuse by Urology Patients and of Information Available on Urological Topics,” BJU International, Vol. 86, No. 3, 2000, pp. 191-194. doi:10.1046/j.1464-410x.2000.00831.x

[15] S. B. McPherson, S. M. Beall, M. L. Greenfield and J. S. Biermann, "Patient Internet Use in a Community Outpatient Orthopaedic Practice,” The Iowa Orthopaedic Jour- 
nal, 2002, Vol. 22, pp. 103-107.

[16] S. B. McPherson, G. J. Gergory, M. L. Greenfield, R. N. Hesinger and J. S. Biermann, "Use of the Internet by Pediatric Orthopaedic Outpatients," Journal of Pediatric Orthopaedics, Vol. 22, No. 2, 2002, pp. 261-264. doi:10.1097/01241398-200203000-00026

[17] B. Makar, D. Quilliot, R. Zarnegar, T. Levan, A. Ayav, L. Bresler, P. Boissel and L. Brunaud, "What Is the Quality of Information about Bariatric Surgery on the Internet?" Obesity Surgery, Vol. 18, No. 11, 2008, pp. 1455-1459.
doi:10.1007/s11695-008-9507-X

[18] M.l Droomers and G. P. Westert, "Do Lower Socioeconomic Groups Use More Health Services, because They Suffer from More Illnesses?” European Journal of Public Health, Vol. 14, No. 3, 2004, pp. 311-313.

[19] R. L. Sudore, et al., "Limited Literacy in Older people and Disparities in Health and Healthcare access," Journal of the American Geriatrics Society, Vol. 54, No. 5, 2006, pp. 770-776. doi:10.1111/j.1532-5415.2006.00691.x 